PROCEEDINGS of the Tenth International Driving Symposium on Human Factors in Driver Assessment, Training and Vehicle Design

\title{
DRIVING SIMULATION AS VIRTUAL REALITY EXPOSURE THERAPY TO REHABIL- ITATE PATIENTS WITH DRIVING FEAR AFTER TRAFFIC ACCIDENTS
}

\author{
Stefanie Schoch ${ }^{1}$, Yvonne Kaussner ${ }^{1}$, A.M. Kuraszkiewicz ${ }^{2}$, S. Hoffmann ${ }^{1}$, P. Markel ${ }^{2}$, \\ R. Baur-Streubel ${ }^{2}$, P. Pauli ${ }^{2,3}$ \\ ${ }^{1}$ Würzburg Institute for Traffic Sciences (WIVW), Veitshöchheim, Germany \\ ${ }^{2}$ Department of Psychology 1 (Biological Psychology, Clinical Psychology, and Psychotherapy), \\ University of Würzburg, Würzburg, Germany \\ ${ }^{3}$ Center of Mental Health, Medical Faculty, University of Würzburg, Würzburg, Germany \\ Email: schoch@wivw.de
}

\begin{abstract}
Summary: Following a traffic accident, up to $30 \%$ of the involved persons suffer from stress related symptoms often coming along with enduring fear of driving. Virtual reality exposure therapy (VRET) offers major advantages for treating anxiety disorders, but with respect to fear of driving it has been hardly investigated so far. In the present study a driving simulator exposure treatment for patients with fear of driving after a traffic accident was developed and evaluated. The therapy followed a standardized manual of 13 sessions including anamnesis, medical examination, two preparative psychotherapy sessions, five virtual reality exposure (VRE) sessions, a final behavioral avoidance test in real traffic with a driving instructor, a closing session, plus follow-up phone calls after six and twelve weeks. The exposure scenarios were individually tailored to the patients' anxiety hierarchy. 14 patients were treated. Results indicate excellent treatment success. In the final behavioral avoidance test, all patients mastered driving tasks they had avoided before, $71 \%$ showed an adequate driving behavior as assessed by the driving instructor, 93\% could maintain their treatment success until the second follow-up phone call. We conclude that VRET in a driving simulator is a highly promising tool to treat fear of driving. Major advantages are that traffic scenarios are highly controllable, safe and can be designed and presented to perfectly fit the individuals' anxieties.
\end{abstract}

\section{INTRODUCTION}

Between 2006 and 2015, there were about 5.770.000 police-reported traffic crashes in the U.S. each year, in which on an annual average 35.500 people were killed and an estimated amount of 2.354.000 people were injured (NHTSA, 2017). Rates of posttraumatic stress disorders following a traffic accident range from 8 to over 30\% (Maercker, 2003; Wiederhold \& Wiederhold, 2010), and up to $30 \%$ of the affected persons develop other psychopathologies like acute stress reactions, adjustment disorders or specific (isolated) phobias (Blanchard \& Veazey, 2001). These disorders often go along with fear of driving causing patients to avoid driving in general or avoid specific driving situations, like highways or unknown routes (Mayou, Simkin \& Threlfall, 1991; Taylor \& Koch, 1995). In some cases, the fear-inducing situations are coped with overcautious driving that might cause obstruction of traffic. Exposure therapy is considered the method of choice in treating anxiety disorders, whereas virtual reality becomes increasingly famous. Treatment efficacy of VRE has been proven for posttraumatic stress disorder (e.g., Gonçalves et al., 2012) and specific phobias like arachnophobia (e.g., Garcia-Palacios et al., 2002), acrophobia (e.g., Emmelkamp et al., 2001) as well as for fear of flying (e.g., Mühlberger et al., 2001). Prom- 
ising results also exist for panic disorder and social phobia (Meyerbröker \& Emmelkamp, 2010). Direct comparisons of virtual reality vs. in vivo exposure, for instance on the treatment of arachnophobia and acrophobia, revealed that both forms of therapy are similarly effective (Emmelkamp et al., 2002; Michaliszyn et al., 2010). VRET offers major advantages in comparison to in vivo exposure: First, refusal rates are lower and acceptance is higher (Garcia-Palacios et al., 2007), Second, VRET is characterized by high situational and therapeutic control as the relevant exposure situations can be selectively designed and purposefully repeated or prolonged. This is especially relevant for the treatment of driving fear, as VRET allows controlling the surrounding traffic, for example the number of oncoming trucks, realizing an accident that has to be passed, or selecting weather conditions. Third, unwanted or unexpected situations can be avoided and the virtual exposure can be stopped immediately at any time which might be of importance in case of psychological and somatic fear reactions of the patient causing security risks. Fourth, VRET facilitates the standardization of exposure and the possibility to measure multiple fear responses as outcome measures. Fifth, an advantage concerning treatment of driving fear is that there is no need for a co-driver in the simulator which could elsewise be a dysfunctional safety signal. Finally, VRET likely has a cost-effectiveness advantage as a result of less sessions needed and higher acceptance by patients (Wiederhold \& Wiederhold, 2010). Despite these adavantages, studies examining its efficacy are rare and investigated very small sample sizes (e.g. Beck et al., 2007; Walshe et al., 2003; Wiederhold \& Wiederhold, 2010). Specifically, even though they suggested promising effects, VRET effects have not yet been assessed with respect to their impact on real driving behavior as assessed with a behavioral avoidance test. Thus, the main objective of the present study was to develop and evaluate a VRET protocol for fear and avoidance of driving after a traffic accident. VRET was conducted in a high-fidelity driving simulator with a real car mockup. A post-treatment behavioral avoidance test in real traffic was realized to serve as primary outcome measure. Maintenance of treatment success was assessed by six and twelve week follow-up phone calls.

\section{METHODS}

\section{Participants}

In total, 14 patients, 5 male and 9 female with a mean age of 40.36 years $(\mathrm{sd}=8.57 \mathrm{yrs} ; \mathrm{min}=28$ $\mathrm{yrs}, \max =53 \mathrm{yrs}$ ) were treated. Mean duration of driving fear and avoidance of driving was 18.0 months ( $\mathrm{sd}=17.0$ months, $\min =2.5$ months, $\max =49$ months). Six patients were full avoiders, eight avoided specific driving situations only (such as highways or larger urban areas). Eight subjects were diagnosed having posttraumatic stress disorder, six persons met the criteria for Specific Phobia. Inclusion criteria were: age of 18 to 63 years, valid driving license, severe symptoms of fear and avoidance of driving a car (complete avoidance or of specific car driving situations) for at least four weeks; the driving fear and avoidance had to be triggered by a traffic accident or another critical event in traffic; regular driving experience prior to the triggering event; diagnosis of adjustment disorder, specific (isolated) phobia or posttraumatic stress disorder; no or stable therapy with psychoactive drugs in the last four weeks. Exclusion criteria were: alcohol/drug addiction, the influence of alcohol/drugs during the triggering event, suicidal tendencies, a positive pregnancy test (for females only), psychosis or other premorbid mental disorders or comorbidities such as coronary heart disease and epilepsy as well as severe cognitive impairment, or another contraindication to an exposure therapy. 


\section{Design, procedure and treatment}

The treatment was conducted as a weeklong block therapy and consisted of 13 sessions (an anamnesis, two preparative psychotherapy sessions, five VRE sessions, a final behavioral avoidance test in real traffic with a driving instructor, a closing session and two follow-up phone calls). The block therapy was conducted on the basis of a specially prepared treatment manual developed by the authors. To control time effects, spontaneous remissions and possible effects of study-related activities (which were independent of driving exposure, such as anamnesis, medical consultation), participants were randomly assigned to either a waiting group (WG, control group) or a treatment group (TG). For the TG and the WG the treatment started directly the day after the anamnesis or seven days after the anamnesis, respectively. However, due to logistic reasons and in order to facilitate the organization of the study, the last five patients were allocated to TG without randomization according to their individual preferences. In consequence, the TG comprised of nine patients and only five were in the WG.

\section{Driving simulator and virtual environments}

VRET sessions were conducted in the high-fidelity fixed base driving simulator of the Wuerzburg Institute for Traffic Sciences (WIVW, see Figure 1). The visual system is comprised of five image channels that provide nearly a panoramic view with a seamless projection $\left(300^{\circ}\right.$ horizontally, $47^{\circ}$ vertically). Rear view is simulated by an LCD-display in the trunk and a real rearview mirror. Outside mirrors are LCD-displays. The mockup is a real Opel Insignia with automatic transmission. The psychotherapist could accompany the exposure as a passenger or in a separate room next to the simulator (operator room). The operator room is connected to the patient by microphone, allowing to talk to the patients during the drives. There are several displays to watch the driver, the current driving scene and various driving parameters including the drivers' current heart rate in real-time. The software, SILAB ${ }^{\circledR}$ (www.wivw.de), is used to operate the driving simulation. The driving scenarios were specifically designed for this study and tailored to the patients' individual anxiety hierarchy, which was elaborated with the psychotherapist in the preparative sessions. Thus, the scenarios were individually selected for the patients and the sessions so that they were neither the same for the drivers nor the sessions (see Figure 1 for some examples). Before the actual exposure treatment, patients had to familiarize with the simulator in order to reduce possible symptoms of simulator sickness and to practice handling the simulator vehicle (steering, accelerating and braking). For this purpose very easy routes (with no or a reduced number of other simulated vehicles) were driven initially in order to prevent anxiety. In the four exposure sessions the participants experienced scenarios which were specifically tailored to their individual anxiety hierarchy (gradually from lower to upper). In the fourth exposure session routes of the medium and upper level were repeated. All scenarios were programmed to be driven in an infinite loop. The final selection as well as the duration of each drive depended on the current level of anxiety of the individual patient during the sessions.

\section{Outcome measures}

Primary outcome: The primary outcome criterion (behavioral avoidance test) was the patients' behavior in the final real driving test with the driving instructor during at least one driving task 
which they had rated as not manageable (or manageable only with very strong anxiety) in a hypothetical driving test before the treatment. The hypothetical driving test consisted of the following seven questions about driving tasks: "If you had the chance to drive with a driving instructor right now, would you (1) sit behind the wheel? Yes/No; (2) drive around a parking area? Yes/No; (3) drive around the block? Yes/No; (4) drive on a rural road? Yes/No; (5) drive on a main road with two lanes? Yes/No; (6) drive on a highway? Yes/No; (7) drive through an urban area (Wuerzburg, Germany)? Yes/No. In the case of "yes" answers patients rated the level of anticipated anxiety on a 11-point subjective units of distress (SUD) scale (ranging from $0=$ no distress, totally relaxed to $10=$ highest anxiety, loss of control). Relevant driving avoidance was an inclusion criterion and defined as complete avoidance or an anticipated anxiety level of 6 or more in at least one of the seven assessed driving tasks. This hypothetical driving test was repeated for the WG also after the waiting period resp. before starting their treatment. For ethical reasons (e.g. risk of a failure or psychological decompensation without immediate therapy for the WG) no real driving test was offered before the treatment. For the real driving test after the treatment only those tasks that had been avoided in the anamnesis were selected. However, for logistic reasons no more than four tasks could be realized. Patients were considered to be successfully treated (treatment responders) if they mastered at least one of those primarily avoided tasks in the final behavioral avoidance test.

Outcomes describing VRET sessions: In addition to the number and the duration of the VR drives, anxiety and habituation during the VRET sessions were determined by ratings on the SUD scale. For the ratings both the maximum rating per drive and the rating before stopping the drive were considered and averaged over the drives per session.

Secondary and exploratory outcomes: The secondary criterion "driving performance in the behavioral avoidance test" was based on assessments by the driving instructor on an ordinal rating scale, commonly used for official driving tests to evaluate driving ability in Germany (BrennerHartmann, 2002). It consists of four categories: 0 : normal, 1: slightly conspicuous, 2: substantially conspicuous, 3 : severely conspicuous. A rating of 0 or 1 was defined to indicate an adequate driving performance.

Another secondary outcome criterion was "maintenance or extension of treatment success" as assessed by the follow-up phone call twelve weeks after the treatment. The criterion "maintenance" was met if at least one task mastered successfully in the behavioral avoidance test was still performed actively in patients' everyday life according to their self-report, the criterion "extension" referred to success in additional tasks avoided before treatment and not mastered in the behavioral avoidance test.

Evaluation of the therapy by the patients: Twelve weeks after the treatment the participants received a semi-standardized questionnaire for written evaluation of the therapy, which they were asked to send back by mail. Inter alia, it included a general assessment in terms of a grade and the possibility to express their opinion in their own words.

\section{RESULTS}

Primary outcome: The seven driving tasks assessed before (hypothetically) and after treatment (practically) revealed considerable decrease of avoidance behavior following VRET. After 
treatment, $100 \%$ of the participants were able to sit behind the wheel (vs. $86 \%$ before treatment), $100 \%$ were able to drive around a parking area (vs. $71 \%$ before), and $100 \%$ were able to drive around the block (vs. $57 \%$ before), $93 \%$ were able to drive on a rural road (vs. $36 \%$ before), $79 \%$ were able to drive on a main road with two lanes (vs. $7 \%$ before), $43 \%$ were able to drive on a highway (vs. $0 \%$ before), and 57\% were able to drive through an urban area (Wuerzburg, Germany; vs. 14\% before). After VRET, all patients mastered at least one of their primarily avoided tasks in the behavioral avoidance test, 13 of the 14 patients even completed all of the offered driving tasks. None of the five patients of the WG reported any improvement/recovery during the waiting period for any of their initially relevant tasks. The same holds true for all other outcome measures. Since randomization was not fully maintained results are not presented separately.

VRET sessions: On average, three drives were completed per exposure session $(\min =2, \max =$ 4) with a mean duration of 19.6 minutes per drive $(\mathrm{sd}=11.6, \min =5$, $\max =60$ minutes $)$. SUDratings indicate that VR drives elicited considerable anxiety which generally decreased between sessions. For example, SUDs of 6 or higher were reached by $93 \%$ of the participants in the first session, and by $79 \%, 86 \%, 71 \%$ resp. in the following sessions. Similarly, mean maximum SUD scores of each session decreased over sessions (Friedman test $X^{2}=14.85 ; p=.002$; see Figure 2) The mean maximum SUD in the third session, when scenarios of upper hierarchy level were driven, was approximately as high as in the first session. Within sessions analyses indicated successful habituation of anxiety in all patients, i.e. fear ratings decreased significantly at least 2 points on the SUD Scale during all the virtual drives and within each session (Wilcoxon signed rank test: session $1: Z=-3.30 ; p=.001$; session $2: Z=-3.30 ; p=.001 ; \operatorname{session} 3: Z=-3.30 ; p=$ .001 ; session $4: Z=-3.30 ; p=.001$; see also Figure 2 ).

Secondary and exploratory outcomes: According to the driving instructor, 10 of the 14 patients (71\%) showed an adequate driving performance during the behavioral avoidance test (4 normal, 6 slightly conspicuous). Three patients drove substantially conspicuous, one even severely conspicuous. Mostly, driving performance was rated as being conspicuous because of an anxious driving style (driving too slowly, unsteady longitudinal control, overcautious securing behavior). 13 out of $14(93 \%)$ patients could maintain or even extend their treatment success until the follow-up phone call resp. for at least 12 weeks.

Evaluation: Twelve weeks after therapy patients received an evaluation questionnaire which was sent back by eleven persons $(79 \%)$. The general assessment $(1=$ very good, $2=$ good, $3=$ satisfying, $4=$ sufficient, $5=$ defective, $6=$ insufficient/failed) resulted in an average rating of good to very good $(\mathrm{m}=1.63 ; \mathrm{sd}=0.89)$. The majority of patients were overwhelmed by their success; tears of joy appeared after the behavioral avoidance test, and feelings of great happiness were reported: "I appreciate it so much that I have been able to participate. I would have never agreed to an exposure directly in real traffic.", "Driving in the simulator felt so real. It helped a lot to reduce my fear and to gain security again."

\section{CONCLUSIONS}

Overall, this study indicates that VRET with driving simulation is a highly efficient tool to treat patients with driving fear and avoidance. Treatment success was evident in post-treatment real driving and was maintained at least for three months. VRET for driving fear and avoidance has 
crucial advantages compared to in vivo exposure since virtual traffic scenarios can be selectively designed and presented to perfectly fit the individuals' anxieties without real danger and without having to search for the specific situations in real traffic. However, further studies are needed to evaluate VRET efficacy for driving fear with a randomized controlled trial, preferably with an active control group. For a widespread usage it is also important to evaluate the necessary degree of individualization for the driving scenarios (e.g. a package of typical scenarios) and the necessary configuration stages of the driving simulator. In addition, a software package with a user friendly interface is needed as well as a verification of treatment efficacy for in-patient and outpatient settings.
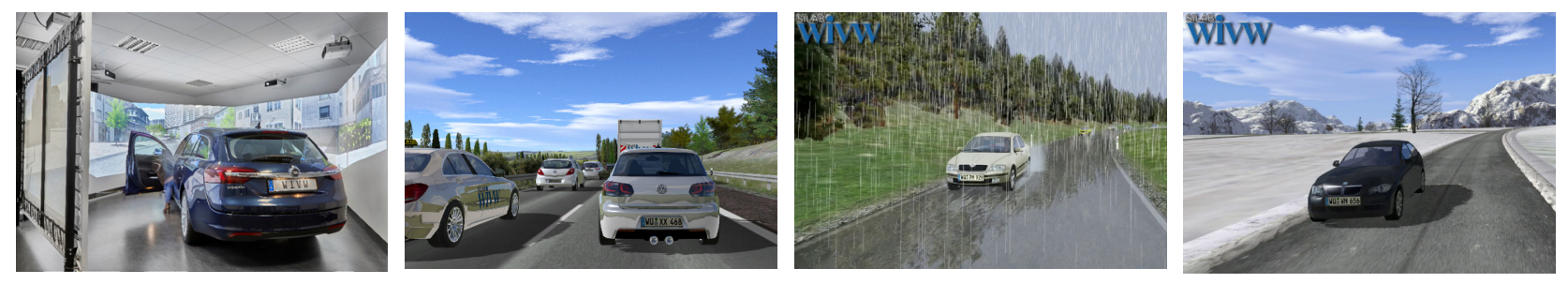

Figure 1: Static driving simulator of WIVW and exemplary virtual scenarios.

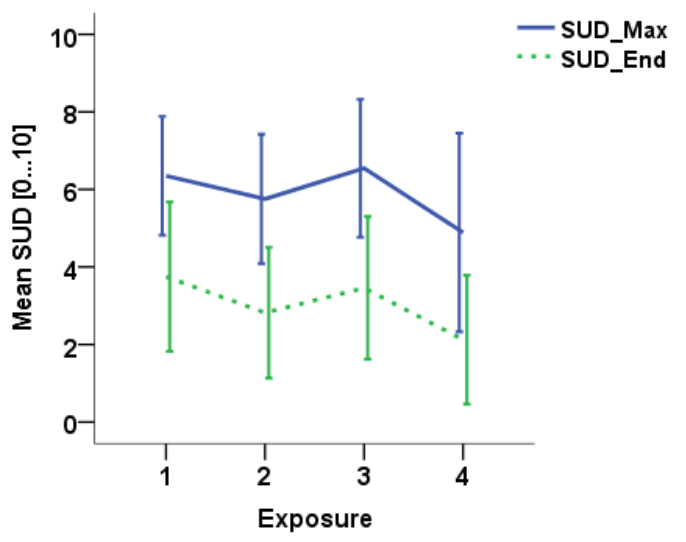

Figure 2: Mean maximum and end SUD score (+/- 1 SD given as error bars) over the four exposure sessions.

\section{ACKNOWLEDGMENTS}

The study was funded by the German statutory accident insurance (DGUV; grant number FR232). DGUV was mainly responsible for recruiting the patients. The authors are fully responsible for the scientific content of this paper. We are grateful to the staff of the driving school Kwiotek and the Medical Study Center Würzburg for their assistance.

\section{REFERENCES}

Beck, J. G., Palyo, S. A., Winer, E. H., Schwagler, B. E., \& Ang, E. J. (2007). Virtual reality exposure therapy for PTSD symptoms after a road accident: An uncontrolled case series. Behavior Therapy, 38, 39-48.

Blanchard, E. B. \& Veazey, C. H. (2001). Mental disorders resulting from road traffic accidents. Current Opinion in Psychiatry, 14 (2), 143-147. 
Brenner-Hartmann J (2002). Durchführung standardisierter Fahrverhaltensbeobachtungen im Rahmen der medizinisch-psychologischen Untersuchung (MPU). BDP-Kongress für Verkehrspsychologie, Regensburg. http://www.mpu-beratung- vorbereitung.de/mpu_beratung_mpu_vorbereitung_sonstiges_fvbbrenner.pdf

Emmelkamp, P. M. G., Bruynzeel M., Drost L. \& van der Mast C. A. P. G. (2001). Virtual reality treatment in acrophobia: a comparison with exposure in vivo. Cyberpsychology and Behavior, 4 (3), 335-339.

Emmelkamp, P. M. G., Krijn, M., Hulsbosch, A. M., de Vries, S., Schuemie, M. J. \& van der Mast, C. A. P. G. (2002). Virtual reality treatment versus exposure in vivo: a comparative evaluation in acrophobia. Behavior Research and Therapy, 40 (5), 509-16.

Garcia-Palacios, A., Botella, C., Hoffman, H. \& Fabregat, S. (2007). Comparing Acceptance and Refusal Rates of Virtual Reality Exposure vs. In Vivo Exposure by Patients with Specific Phobias. Cyberpsychology and Behavior, 10 (5), 722-724.

Garcia-Palacios, A., Hoffman, H., Carlin, A., Furness, T. A. III, \& Botella, C. (2002). Virtual reality in the treat-ment of spider phobia: A controlled study. Behaviour Research and Therapy, 40, 983-993.

Gonçalves, R., Pedrozo, A. L., Coutinho, E. S. F., Figueira, I. \& Ventura, P. (2012). Efficacy of Virtual Reality Exposure Therapy in the Treatment of PTSD: A Systematic Review. PLOS ONE, 7 (12), e48469, 1-7.

Maercker, A. (2003). Therapie der posttraumatischen Belastungsstörungen (2nd ed.). Berlin, Heidelberg: Springer-Verlag.

Mayou, R., Simkin, S. \& Threlfall, J. (1991). The effects of road traffic accidents on driving behaviour. Injury, 22 (5), 365-368.

NHTSA (National Highway Traffic Safety Administration) (2017). Summary of Motor Vehicle Crashes (Final Edition). Traffic Safety Fact Sheet. dot: HS 812376.

Meyerbröker, K. \& Emmelkamp, P. M. G. (2010). Virtual reality exposure therapy in anxiety disorders: A systematic review of process-and-outcome-studies. Depression and Anxiety, 27, 933-944.

Michaliszyn, D., Marchand, A., Bouchard, S., Martel, M.-O., \& Poirier-Bisson, J. (2010). A randomized, controlled clinical trial of in virtuo and in vivo exposure for spider phobia. $C y$ berpsychology, Behavior and Social Networking, 13, 689-695.

Mühlberger, A., Herrmann, M. J., Wiedemann, G., Ellgring, H. \& Pauli, P. (2001). Repeated exposure of flight phobics to flights in virtual reality. Behaviour Research and Therapy, 39, 1033-1050.

Walshe, D. G., Lewis E. J., Kim, S. I., O’Sullivan, K. \& Wiederhold, B. K. (2003). Exploring the Use of Computer Games and Virtual Reality in Exposure Therapy for Fear of Driving Following a Motor Vehicle Accident, Cyberpsychology \& Behavior, 6 (3), 329-334.

Wiederhold, B. K. \& Wiederhold, M. D. (2010) Virtual Reality Treatment of Posttraumatic Stress Disorder Due to Motor Vehicle Accident. Cyberpsychology, Behavior and Social Networking, 13 (1), 21-27. 